\title{
Design and Implementation of Microcontroller Based Calculator
}

\author{
Adedoyin M. A. ${ }^{1}$, Olopade o.I. ${ }^{2}$, Shoewu O. O. ${ }^{3}$, Ogunlewe A.O. ${ }^{4}$ \\ *Corresponding author: Shoewu O. O \\ Department of Electronic and Computer Engineering, \\ Lagos State University, Lagos Nigeria. \\ E-mail: engrshoewu@yahoo.com
}

Received: April 2, 2013, Accepted: April 23, 2014, Published: April 24, 2014.

\begin{abstract}
This paper focused on design and implementation of a microcontroller based calculator which will perform simple arithmetic using a calculator keypad and a 16x2 Liquid Crystal Display (LCD). The program is limited to single digit input and double-digit results. This allows the program for the arithmetic operations to be simple, while the same principles can be extended to multi-digit calculations.
\end{abstract}

Keywords: Microcontroller, Calculator, keypad, LCD display.

\section{INTRODUCTION}

Advancement in technology has led to building electronic devices with simple circuit. Introduction of microcontroller has made designing of electronic devices circuit simpler. A computer on a chip is known as microcontroller. It is essential for the operation of devices such as mobile phones, video cameras, electrical appliances and most self-contained electronic systems. Microcontroller has the following elements: memory, central processing unit, ports, bus, serial communication, etc.

Memory: Program and data are stored in the memory. Central Processing Unit (CPU) has capability to multiply, divide, subtract, and move its contents from Memory locations which are called registers. Registers are memory locations whose role is to perform various mathematical operations or any other operations with data wherever data can be found. Bus is a connection between memory and CPU-the path through which data goes from one block to another. Physically, it represents a group of 8,16 , or more wires. There are two types of buses: address and data buses. The first one has many lines as the amount of memory to address, and the other one is as wide as data. First one transmits address from CPU memory, and the second connects all blocks inside the microcontroller. Ports have several memory locations whose one end is connected to the data bus, and the other has connection with the output lines on the microcontroller which can be seen as pins on the electronic component. There are several types of ports: input, output or bidirectional ports. When working with ports, first of all it is necessary to choose which port one needs to work with, and then to send data to, or take it from the port. When working with it the port acts like a memory location. Something is being written into or read from it, and this could be noticed on the pins of the microcontroller. Watchdog is a free-run counter where a program needs to write a zero in every time it executes correctly. Analog to digital converter (ADC) is responsible for converting an information about some analog value to a binary number and follow it through to a CPU block so that CPU block can further process it. Finally, the microcontroller is completed, and all need to be done is to assemble it into an electronic component where it will access.

\section{Theory of Design}

The circuit for a calculator which will perform simple arithmetic operations in the PIC 16F887 Microcontroller, using a calculator keypad and 16X2 LCD display, is shown in Figure1. The keypad has 16 keys: 10 numeric buttons, 4 arithmetic operations, equals and clear. The results obtained are displayed on the LCD display.

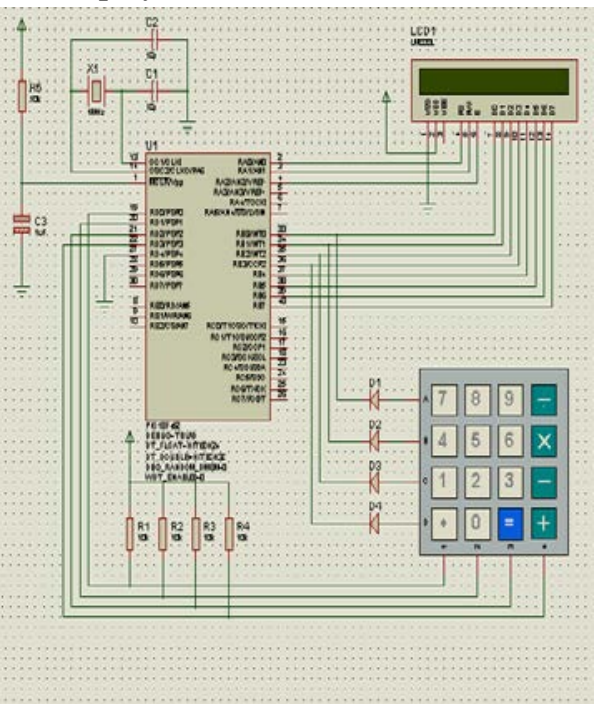

Figure 1: Calculator Circuit Diagram 


\section{The calculator operates as follows:}

- In order to perform a calculation, press a number key, followed by an operation key, then another number and then equals.

- The calculation and result are displayed. For the divide operation, the result is displayed as result and remainder.

- The clear key erases the current display, and a new calculation can be entered. If an invalid key sequence is entered, the program should be restarted.

The calculation routine uses the operation input code to select the required process: add, subtract, multiply or divide. The binary result of the calculation is passed to a routine to convert it into BCD, then ASCII, and send it to the display. The result of the divide, being a single digit result and remainder, is sent direct to the display. The clear operation sends a command to the display to clear the last set of characters.

\section{Design Overview}

The design overview is divided into two, hardware and software.

\section{A.Hardware design}

The Hardware Design is into the modules for easy design, analysis and integration.

- Power Supply unit

- Control Unit

- Input Unit

- Output Unit

Each module was design individually and then joined together to form a calculator. The different modules are controlled by a series of C-programs linked into the control unit. Figure 1. shows the system block diagram.

\section{Power Supply Unit}

power supply unit provides the electronic circuit with necessary direct voltages and current with low level of A.C ripples and good stability. The power supply comprises of a transformer, a rectifier, a filter and a regulator.

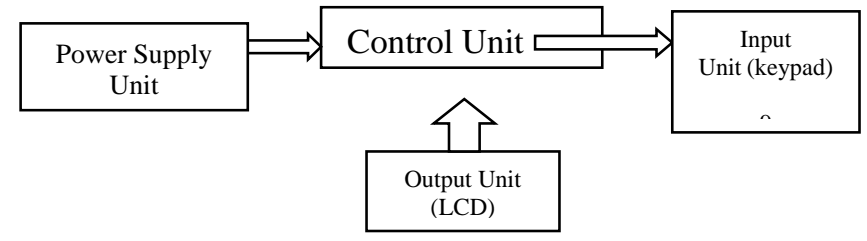

Figure 2. Block Diagram of the calculator

\section{Input Unit}

The $4 \times 4$ keypad used in this design serves as an input unit through which the user communicates

with the system.

\section{Output Unit (LCD)}

The output unit is the LCD the calculator is a $16 \times 2$ LCD. It has a back-ground light which enhances readability in low light conditions.

\section{Control Unit}

The microcontroller is CPU of the calculator. It gives instructions, accepts inputs through the keypad, process it, and displays the processed output through the LCD.

\section{B.Software Design}

In the software design, the program outline and flow diagram show how the system works. It carefully illustrates the flow of instructions from one step to the other during operation.

\section{Program outline for implementing the calcultor}

The program outline used to implement the the calculator is shown below:

1. Single digit calculator produces two digit results.

2. Hardware: x12 keypad, 2x16 LCD, P16F887 MCU

3. MAIN

4. Initialise

5. $\quad$ PortC $=$ keypad

6. $\mathrm{RC} 0-\mathrm{RC} 3=$ output rows

7. RC4 $-\mathrm{RC} 7=$ input columns

8. $\quad$ PortD $=\mathrm{LCD}$

9. $\mathrm{RD} 1, \mathrm{RD} 2=$ control bits

10. $\mathrm{RD} 4-\mathrm{RD} 7=$ data bits

11. CALL Initialise display

12. Scan Keypad

13. REPEAT

14. CALL Keypad input, Delay 50ms for debounce

15. CALL Keypad input, Check key released

16. IF first key, load Num1, Display character and restart loop

17. IF second key, load sign, Display character and restart loop

18. IF third key, load Num2 Display character and restart loop

19. IFfourth key, CALL Calculate result

20. IF fifth key, Clear display

21. ALWAYS 


\section{SUBROUTINES}

23. Included LCD driver routines

24. Initialise display

25. Display character

26. Keypad Input

27. Check row A, IF key pressed, load ASCII code

28. Check row B, IF key pressed, load ASCII code

29. Check row C, IF key pressed, load ASCII code

30. Check row D, IF key pressed, load ASCII code

31. ELSE load zero code

32. Calculate result

33. IF key = ' + ', Add

34. IF key = ‘-‘, Subtract

35. IF key $=$ ' $x$ ', Multiply

36. IF key $=$ ' ', Divide

37. Add Add Num1 + Num2

38. Load result, CALL Two digits

39. Subtract Subtract Num1 - Num2

40. IF result negative, load minus sign, CALL Display character

41. Load result, CALL Display character

42. Multiply

43. REPEAT

44. Add Num1 to Result

45. Decrement Num2

46. UNTIL Num2 $=0$

47. Load result, CALL Two digits

48. Divide

49. REPEAT

50. Subtract Num2 from Num1

51. Increment Result

52. UNTIL Num1 negative

53. Add Num2 back onto Num1 for Remainder

54. Load Result, CALL Display character

55. Load Remainder, CALL Display character

56. Two digits

57. Divide result by 10, load MSD, CALL Display character

58. Load LCD, CALL Display character

\section{Construction of the Project}

The system is constructed on the board after the circuit has been analysed and simulated in proteus ISIS Professional 7.8 portable simulation software.

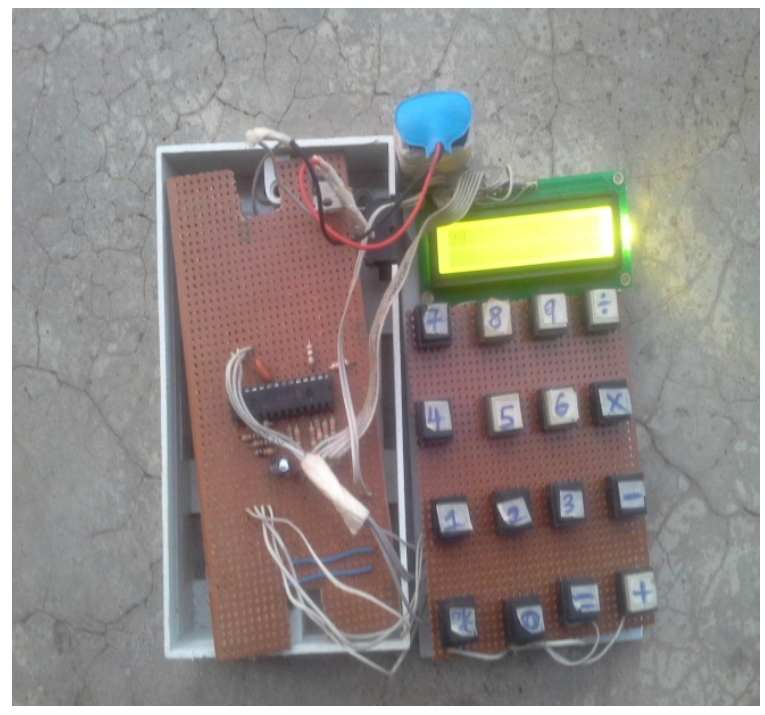

Figure 4: The system construction

\section{Implementation and Testing}

The system is successfully implemented based on the system design, Microcontroller PIC 16F783A was programmed with C-language and compiled using CCS_PCWH_V3.203 PIC C Compiler Series of programs were written and simulated using PIC simulator IDE and MPLAB before the working program was finally achieved and then transferred to the microcontroller chip using PICCALL software / hardware parallel port interface PIC programmer. The actual prototype implementation, simulations were carried out to test if the codes were working correctly. A sampled result was obtained and this was compared with that of other calculators and the results were the same.

\section{CONCLUSION}

There is need for a portable, reliable, low cost and faster means of calculation with simple design. This study designed and implemented a Microcontroller based calculator for easy and speedy calculation. The Microcontroller PIC 16F887 was programmed with C-language and compiled using CCS_PCWH_V3.203 PIC C Compiler and proteus ISIS Professional 7.8 portable simulation software. Results of the calculator were found to agree with the other calculators. 


\section{REFERENCES}

1. Martin Bates (2006). Interfacing PIC Microcontrollers, Embedded Design by Interactive Simulation.

2. Electronics Projects with Microcontroller www.jap.hu/electronics.html

3. Introduction to PIC Programming www.quasare/eletronics.com
4. PIC16F887 Datasheet - http://ww1.microchip.com/ downloads/en/devicedoc/35007b.pdf

5. Nebojsa Matic and Dragan Andric (2010) PIC Microcontroller for beginners

6. Programming PIC16F873A Micro-controllers www.ubasics.com.

Citation: Shoewu O. O, et al (2014). Design and Implementation of Microcontroller Based Calculator. J. of Computation in Biosciences and Engineering. V1I2.

Copyright: (C) 2014 Shoewu O. O . This is an open-access article distributed under the terms of the Creative Commons Attribution License, which permits unrestricted use, distribution, and reproduction in any medium, provided the original author and source are credited. 